Franziska Liechti ' , Luis Falcato ${ }^{1}$, Kenneth M. Dürsteler-Mac Farland 1,2,

Wulf Rössler ${ }^{1}$, Rudolf Stohler ${ }^{1}$

1 Psychiatrische Universitätsklinik Zürich, Sektor West und Zentrale Sozial-

psychiatrische Dienste

2 Psychiatrische Universitätsklinik Basel, Bereich Abhängigkeitsstörungen

\title{
Psychosoziale Charakteristika, Methadondosierung und Heroinkonsum in ambulaten Methadonbehandlungen: ein Matched-pair-Design
}

Der Nachweis, dass Methadonbehandlungen bezüglich der Reduktion des Heroinkonsums, des Mortalitäts- und Morbiditätsrisikos und des Delinquenzverhaltens effektiv sind, ist in einer Vielzahl von Studien erbracht worden (Ball \& Ross 1991; Farrell et al. 1994; Bertschy 1995; Marsch 1998; Ward et al. 1999). Die Frage nach der bestmöglichen Ausgestaltung einer Methadonbehandlung wird jedoch weiterhin kontrovers diskutiert.

Es wurde gezeigt, dass die Höhe der Methadondosierung einen wesentlichen Einfluss auf den Behandlungsverlauf hat und dass die adäquate Methadondosierung einer der wichtigsten Prädiktoren für den Erfolg einer Methadonbehandlung ist (Ball \& Ross 1991; Caplehorn et al. 1993; Strain et al. 1999). In neueren Behandlungsrichtlinien werden Methadondosierungen im Bereich zwischen 60 und $120 \mathrm{mg} /$ Tag als angemessen angegeben (Parrino 1993; American Psychiatric Association 1995; Department of Health et al. 1999).

Für Behandlungen, die im Kanton Zürich zwischen 1995 bis 1997 begonnen wurden, liegt die durchschnittliche Methadondosis nach einem Jahr bei $54 \mathrm{mg} /$ Tag. Viele Behandelnde setzen dann höhere Methadondosen ein, wenn Patienten folgende Eintrittsmerkmale aufweisen: höheres Alter, schweizerische Nationalität, Arbeitslosigkeit, Injizieren des Heroins sowie zusätzlicher Gebrauch von Kokain oder Benzodiazepienen (vgl. Tab. 1, erste und zweite Spalte). Diese Behandlungspraxis erschwert die Vergleichbarkeit von Behandlungsverläufen zwischen unterschiedlichen Dosisgruppen.

Wir präsentieren hier einen Vergleich der Entwicklung des Heroinkonsums in unterschiedlich dosierten Methadonbehandlungen mit einer Dauer von mindestens $2 \frac{1}{4}$ Jahren. Die Analyse beruht auf dem routinemässigen Monitoring der Methadonbehandlungen im Kanton Zürich, das seit 1992 fortlaufend durchgeführt wird (zum Erhebungsin- strument vgl. Olgiati et al. 1992). Die Vergleichbarkeit der untersuchten Dosis-Gruppen wird durch die Anwendung eines speziellen Samplingverfahrens (Matched-pair-Design) im Nachhinein sichergestellt. Wir können dadurch eine quasi-experimentelle Untersuchung des zeitlichen Verlaufs des Heroinkonsums in Methadonbehandlungen durchführen und so den direkten Effekt der Methadondosis auf den verbleibenden Heroinkonsum während der Behandlung isolieren.

\section{Datenbasis und Methode}

Die Auswertungen basieren auf 723 Methadonbehandlungen mit einer Mindestdauer von 27 Monaten und einer durchschnittlichen Dosierung von $\leq 50 \mathrm{mg} / \mathrm{Tag}$ bzw. $\geq 80 \mathrm{mg}$ / Tag, die zwischen 1995 und 1997 im Kanton Zürich begonnen wurden. Pro Person gelangt höchstens eine Behandlung in die Analyse.

Die Gruppenzuteilung erfolgt aufgrund des Mittelwerts der halbjährlich erhobenen Dosisangaben zwischen sechs Monaten nach Beginn und drei Monaten vor Abschluss der Behandlung. In Anlehnung an Strain et al. (1999) werden Durchschnittsdosen $\leq 50 \mathrm{mg} /$ Tag als "tief" und solche $\geq 80 \mathrm{mg} /$ Tag als ,hoch" bezeichnet.

Der Heroinkonsum während der Behandlung wird zu Behandlungsbeginn $\left(t_{0}\right)$, nach sechs Monaten $\left(t_{1}\right)$, nach 12 Monaten $\left(t_{2}\right)$, nach 18 Monaten $\left(t_{3}\right)$ und nach 24 Monaten $\left(t_{4}\right)$, in den Kategorien „kein Konsum“ (0) "gelegentlicher Konsum” (1) und „täglicher Konsum“ (2), jeweils für das Zeitfenster der letzten 30 Tage erhoben.

\section{Matched-pair-Design}

Um Einflüsse von Eintrittsmerkmalen und verschiedenen Einrichtungstypen auf die Dosisfindung und den Behandlungsverlauf $\mathrm{zu}$ kontrollieren, wird ein 
Matched-pair-Subsample gezogen. D.h. für jede behandelte Person der Hochdosis-Gruppe wird die Kombination der relevanten Eintrittsmerkmale ermittelt und dann aus allen tief dosierten Behandlungen jeweils zufällig ein übereinstimmender Fall ausgewählt. Auf diese Weise wird erzielt, dass sich die beiden Dosisgruppen in den "gepaarten" Merkmalen nicht unterscheiden.

Folgende Variablen wurden kontrolliert: a) Geschlecht, b) Alter bei Behandlungsbeginn (Quartile, Grenzen bei 27,31, 36), c) Nationalität (es wurden nur Schweizer einbezogen), d) Arbeitssituation (Voll- und Teilzeitarbeit, Temporär- und Gelegenheitsarbeit, IV-Bezug), e) Konsum von Heroin, Benzodiazepinen oder Kokain 30 Tage vor Behandlungseintritt $(1=$ Konsum, $0=$ kein Konsum $), f)$ intravenöser Drogenkonsum ( $1=$ jemals injiziert, $0=$ nie injiziert $), g$ ) Einrichtungstypen (Privatpraxen $\leq 12$ Methadonbehandlungen, Privatpraxen $>12$ Methadonbehandlungen, Drop-in, Zokl, andere Institutionen).

Das mittels des beschriebenen Matching-Verfahrens gezogene Subsample besteht aus 56 hoch und 56 tief dosierten Behandlungen.

Für die Berechnung der Signifikanz von Zusammenhängen und Mittelwertsunterschieden kamen $\mathrm{Chi}^{2}$ - und t-Tests zur Anwendung. Vergleiche von Eintritts- und Verlaufsdaten wurden mit dem Wilcoxon-Rangsummen-Test für abhängige Stichproben durchgeführt.

\section{Ergebnisse}

In Tabelle 1 sind die im Matching-Verfahren kontrollierten Variablen der hoch und tief dosierten Gruppe für Sample und Subsample aufgeführt. Wir stellen fest, dass sich die hoch und tief dosierten Methadonbehandlungen unseres Fallregisters in ihren Eintrittsmerkmalen unterscheiden (vgl. Tabelle 1, die beiden Spalten unter "Sample").

In Abbildung 1 sind die Mittelwerte des dreistufig erfassten Heroinkonsums vor Behandlungsbeginn und während der Behandlung dargestellt. Der Wilcoxon-Rangsummen-Test für abhängige Stichproben ergibt für beide Patientengruppen zu jedem Messzeitpunkt einen gegenüber dem Heroinkonsum vor der Methadonbehandlung signifikant reduzierten Konsum $(p<0,05)$. In der Hochdosisgruppe setzt sich die Reduktion des Heroinkonsums nach sechs Monaten fort, wohingegen dies in der Tiefdosisgruppe nicht der Fall ist.

Zwischen der Gruppe der tief und der hoch dosierten Patienten besteht auf Grund des oben beschriebenen Verlaufs während der Methadonbehandlung, nach zwei Jahren ein signifikanter Unterschied bezüglich des verbleibenden Heroinkonsums (vgl. Abb. 2). Während in der tief dosierten Gruppe $66,1 \%$ der behandelten Personen Heroinkonsum angeben, sind es in der hoch dosierten Gruppe $46,4 \%$ $(\mathrm{p}<0,05)$.

Tabelle 1. Soziodemographische Charakteristika, Drogenkonsum bei Behandlungsbeginn und Einrichtungstyp (wenn nicht anders angegeben, sind die Werte in Prozent)

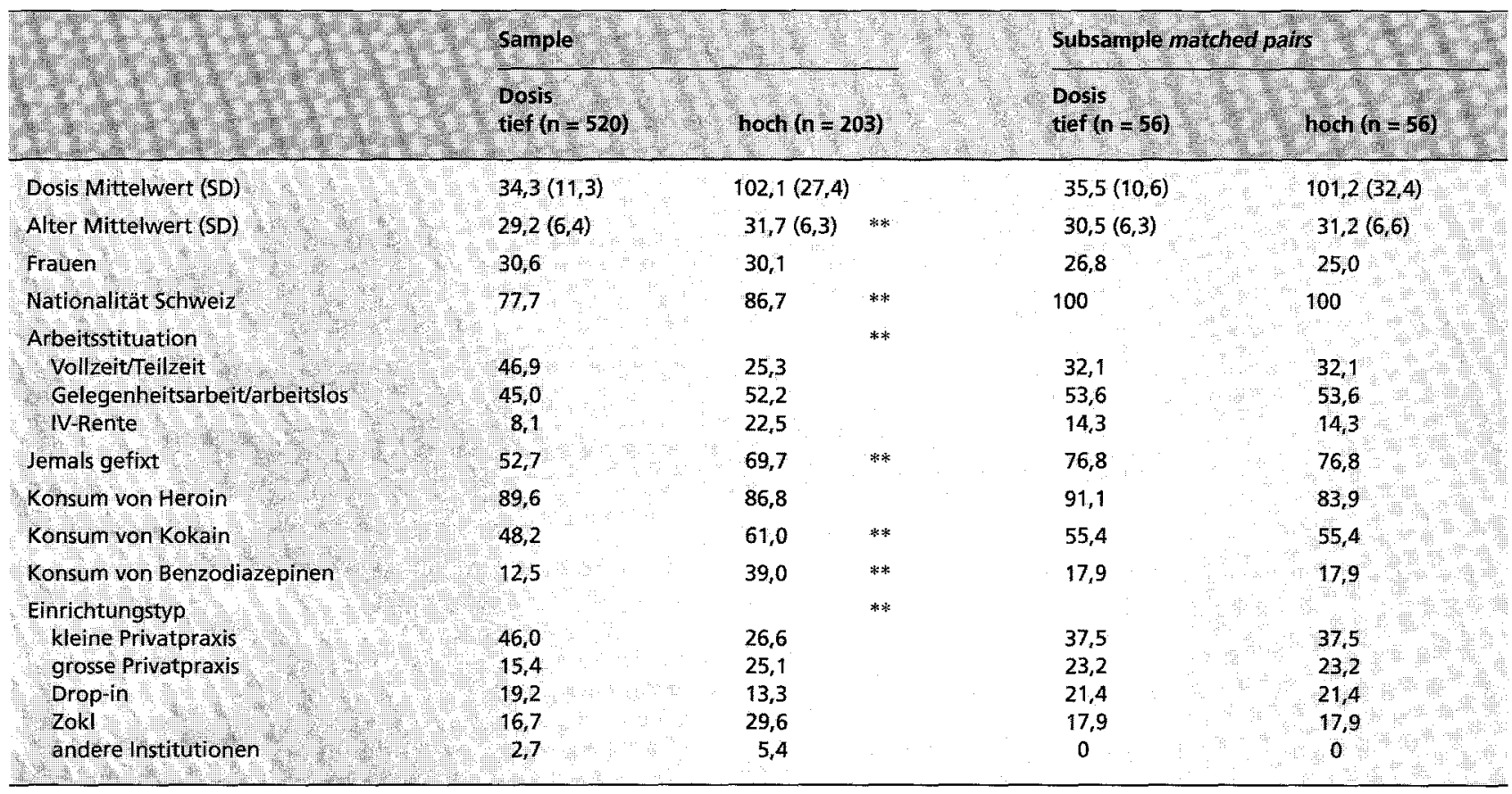

** Der Unterschied zwischen der hoch und tief dosierten Gruppe ist signifikant $(p<0,01)$. 


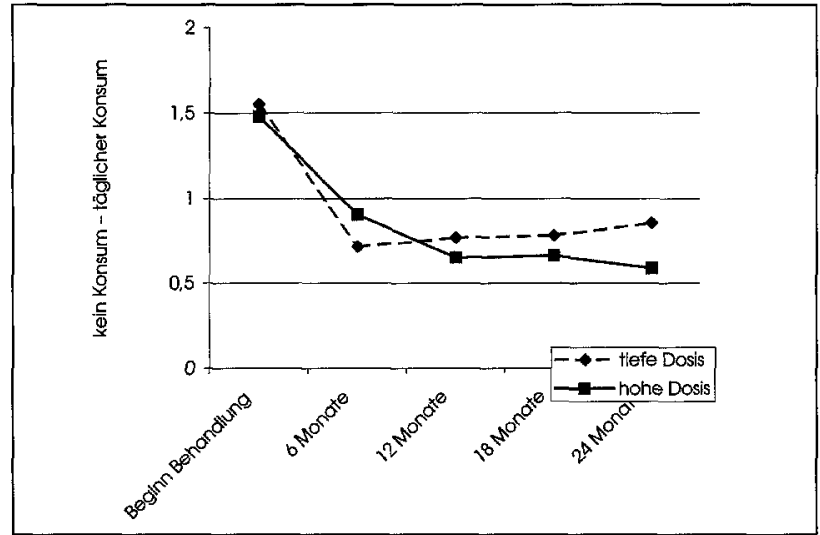

Abbildung 1 Methadondosierung und Heroinkonsum im Behandlungsverlauf $(n=112)$

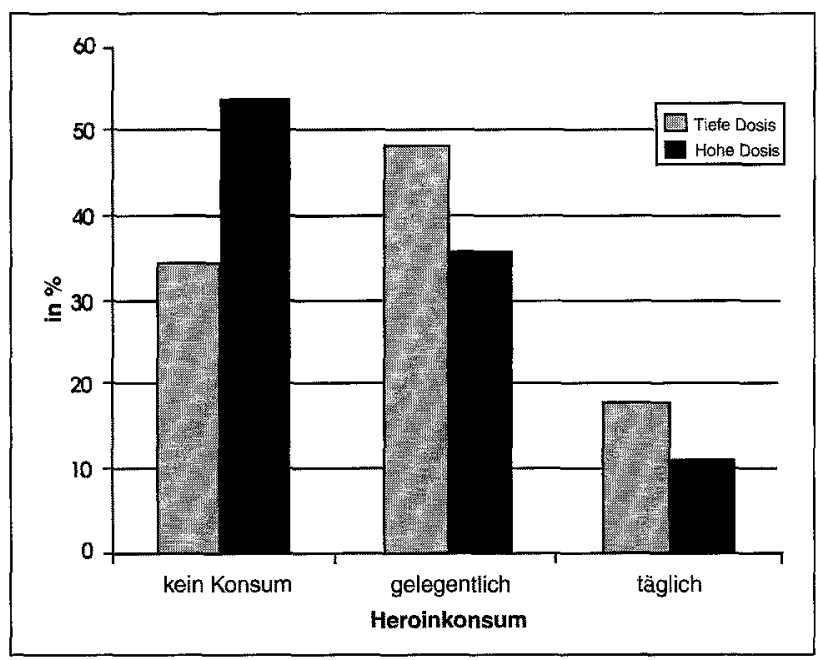

Abbildung 2 Methadondosierung und Heroinkonsum zwei Jahre nach Behandlungsbeginn ( $n=112)$

\section{Diskussion}

Die möglichst vollständige Reduktion des Heroinkonsums ist ein primäres Ziel von Methadonbehandlungen. Das Fortbestehen eines Konsums ist, vor allem bei Injektion des Heroins, mit einem erhöhten Risiko assoziiert, HIV, Hepatitiden oder andere Infektionskrankheiten zu akquirieren. Ebenfalls behindert es das Sistieren von delinquentem Verhalten.

Die kontrollierte Analyse der Daten zu den Methadonbehandlungen im Kanton Zürich zeigt, dass eine höhere Methadondosis mit einer stärkeren Reduktion des Heroinkonsums assoziiert ist. Wir können somit nachweisen, dass ein direkter Zusammenhang zwischen Methadondosierung und Reduktion des Heroinkonsums in nicht experimen- tellen Methadonbehandlungen gezeigt werden kann. Der Zusammenhang wird allerdings erst offensichtlich, wenn mit Hilfe eines Matched-pair-Designs - für unterschiedliche Eintrittsmerkmale und Behandlungssettings korrigiert wird. Das „Verschwinden“ des Zusammenhangs ausserhalb quasiexperimenteller Bedingungen verweist darauf, dass das Ausmass des Heroinkonsums im Verlaufe einer Methadonbehandlung durch viele weitere Einflussfaktoren bedingt wird. Dass solche multivariaten Zusammenhänge lediglich kontrolliert, nicht jedoch in das erklärende Modell einbezogen wurden, stellt eine Limitierung dieser Studie dar.

Unsere Ergebnisse legen nahe, dass es bei ungünstig verlaufenden Methadonbehandlungen aussichtsreich ist, die Effektivität der Behandlung bezüglich Heroinkonsum durch eine Dosiserhöhung zu steigern.

Weshalb wird nicht häufiger mit höheren Methadondosen behandelt? Unsere Daten zeigen, dass Behandelnde bei der Dosierung von Methadon in der Regel bestimmte Eintrittsmerkmale berücksichtigen. Einige davon, wie der Konsum weiterer Substanzen (Kokain, Benzodiazepine) mögen sinnvoll sein und sind zum Teil durch Forschungsergebnisse gestützt (Saxon et al. 1996; Maddux et al. 1997; Borg et al. 1999; Falcato et al. 1999). Dass bei einer guten Arbeitssituation und anderen Konsumformen als Injizierten tiefer dosiert wird, scheint einer unmittelbar einleuchtenden Logik zu folgen. Diese Behandlungspraxis ist jedoch nicht auf wissenschaftliche Forschungsergebnisse abgestützt.

Der „direkte“ Effekt der Methadondosis ist nur unter kontrollierten Bedingungen feststellbar. In der Realität des Behandlungsalltags erscheint die Reduktion des Heroinkonsums während der Behandlung daher eher als Ergebnis eines komplexen Zusammenspiels multipler Faktoren und weniger als direkte Konsequenz einer adäquaten Dosierung. Möglicherweise zögern Behandelnde auch aus Angst vor einer iatrogenen Chronifizierung, bei Patienten mit einem scheinbar günstigeren Profil adäquat zu dosieren. Solche Bedenken scheinen allerdings in den meisten Fällen unberechtigt und können den Erfolg der Behandlung gefährden (Strain et al. 1999).

Der von Patienten vielfach geäusserte Wunsch, Methadon nur niedrig dosiert zu bekommen, spiegelt vermutlich bis zu einem gewissen Grad die Ängste von Behandelnden wider. Diesen Ängsten muss selbstverständlich Rechnung getragen werden. Es sollte jedoch beiden Seiten bewusst sein, dass die Methadondosis in den meisten Fällen gefahrlos bis $120 \mathrm{mg}$ /Tag gesteigert werden kann. 


\section{Literaturverzeichnis}

American Psychiatric Association (1995). Practice guideline for the treatment of patients with substance use disorder: alcohol, cocaine, opioids. Am J Psychiatry 152 (Suppl.).

Ball J, Ross A (1991). The effectiveness of methadone maintenance treatment. New York: Springer.

Bertschy $G$ (1995). Methadone maintenance treatment: an update. Eur Arch Psychiatry Clin Neurosci 245: 114-24.

Borg L, Broe DM, Ho A, Kreek MJ (1999). Cocaine abuse sharply reduced in an effective methadone maintenance program. J Addict Dis 18: 63-75.

Caplehorn JR, Bell J, Kleinbaum DG, Gebski VJ (1993). Methadone dose and heroin use during maintenance treatment. Addiction 88: 119-24.

Department of Health, Scottish Office Department of Health, Welsh Office Department of Health, Social Services Northern Ireland (1999). Drug misuse and dependence: guidelines on clinical management. Norwich: HMSO.
Falcato L, Eichenberger A, Zimmer Höfler D, Rössler W (1999). Die Methadonverschreibung an Opiatabhängige im Kanton Zürich: Behandlungszahlen, Eintrittsmerkmale und Verlauf. In: Mäder C, Burton-Jeangros C, Haour-Knipe $M$, eds. Gesundheit, Medizin und Gesellschaft. Zürich: Seismo: 332-57.

Farrell M, Ward J, Mattick R, et al. (1994). Methadone maintenance treatment in opiate dependence: a review. BMJ 309: 997-1001.

Maddux JF, Prihoda TJ, Vogtsberger KN (1997). The relationship of methadone dose and other variables to outcomes of methadone maintenance. Am J Addict 6: 246-55.

Marsch LA (1998). The effficacy of methadone maintenance interventions in reducing illicit opiate use, HIV risk behavior and criminality: a meta-analysis. Addiction 93: 515-32.

Olgiati M, Dobler-Mikola A, Uchtenhagen A (1992). Die methadonunterstützte Behandlung im Kanton Zürich. Drogen Alkohol 7: 73-103.

Parrino M (1993). State Methadone Treatment Guidelines. Rockville: U.S. Department of Health and Human Services, Public Health Service Substance Abuse and Mental Health Services Administration, Center for Substance Abuse Treatment.
Saxon AJ, Wells EA, Fleming C, Jackson TR, Calsyn DA (1996). Pre-treatment characteristics, program philosophy and level of ancillary services as predictors of methadone maintenance treatment outcome. Addiction 91: 1197-209.

Strain EC, Bigelow GE, Liebson IA, Stitzer ML (1999). Moderate- vs high-dose methadone in the treatment of opioid dependence: a randomized trial. JAMA 281: 1000-5.

Ward J, Hall W, Mattick RP (1999). Role of maintenance treatment in opioid dependence. Lancet 353: 221-6.

\author{
Korrespondenzadresse \\ Luis Falcato \\ Psychiatrische Universitätsklinik Zürich, \\ Sektor West \\ und Zentrale Sozialpsychiatrische Dienste \\ Sozialpsychiatrische Forschungsgruppe \\ Postfach 1930 \\ CH-8021 Zürich
}

e-mail: sofo@spd.unizh.ch 